\title{
Dynamic loads evaluation for the USV3-PRIDE robotic arm qualification
}

\author{
N. Paletta, M. Di Vice \& M. Belardo \\ C.I.R.A. s.c.p.a., The Italian Aerospace Research Centre, Italy
}

\begin{abstract}
This paper presents the evaluation of the dynamic loads for the qualification of a robotic arm, to be installed on-board the USV3-PRIDE spacecraft.

CIRA is developing a fully reusable unmanned space vehicle, code named USV3-PRIDE, to be launched by VEGA. The system is capable of orbiting, de-orbiting and gliding back to Earth with high manoeuvrability and controllability throughout all flight regimes (i.e. hypersonic re-entry, supersonic, transonic, subsonic) and performing a safe ground landing on a conventional runway by a landing gear system. Such aerospace systems could lead to the new technological frontier to master both new and innovative orbital robotic systems in the near Earth environment, as well as to explore the hypersonic flight regimes necessary to re-enter the earth from above $300 \mathrm{Km}$ altitude to the ground, which represent the same flight levels of the future aerospace transportation systems.

The spacecraft is equipped with a robotic arm which provides the capability to ship a payload and interface with a space tug towards the International Space Station.

During ground operation and flight the spacecraft is subject to different kinds of loads, both static and dynamic. These excitations are from different origins such as operational, aerodynamics or propulsion. In particular, for the mechanical qualification of the robotic arm and the other subsystems, the dynamic environment provides for the most severe conditions in terms of local accelerations.

The dynamic environment taken under consideration includes two types of dynamic analyses: Shock Response Spectrum (SRS) analysis, and Sine equivalent dynamics.

SRS analysis is necessary to calculate the structural local response to shocks and to estimate the maximum dynamic response of a structure. The spacecraft is
\end{abstract}


subject to shock primarily during stage separations, fairing jettisoning and actual spacecraft separation.

Sine equivalent dynamics accounts for sinusoidal excitations affecting the launch vehicle during its powered flight (mainly the atmospheric flight), as well as during some of the transient phases. In the case of VEGA launcher the sine vibration is applied to the USV3-PRIDE base exciting all the sub-systems, including the robotic arm.

Results give guidelines for both the robotic arm design and the mechanical interface to the USV3-PRIDE airframe.

Keywords: dynamic analysis, SRS analysis, Shock Response Spectrum, Sine Equivalent Dynamics, Modal Transient Analysis, Nastran, USV3-PRIDE, robotic arm qualification.

\section{Introduction}

During the last decade CIRA, the Italian Aerospace Research Centre, has been leading different research projects concerning in-flight demonstration of key technologies for re-entry systems in the framework of national and international programs [1-4]. Within the national USV (unmanned space vehicle) program [5], the USV3 project is the current driving project [6]. It was created to achieve a technology driven approach, i.e. the design of a system with available technologies with high TRL or COTS (Component Off-The-Shelf) in order to fulfil a set of high level requirements. In this approach the research and development activities are reduced in favour of system engineering effort.

USV3 has the challenging objective to demonstrate the system capability to execute a re-entry flight with a winged body from LEO (Low Earth Orbit), after a number of prescribed revolutions and to safely land on a conventional runway by means of a conventional landing gear system (Figure 1). The vehicle, intended as a technology demonstrator, is currently in the preliminary design stage, after the development of a conceptual design entirely performed by CIRA researchers and engineers [7]. The spacecraft, to be launched by VEGA, is equipped with a robotic arm under development in the framework of a cooperation project, named SAPERE STRONG, funded by the Italian Ministry of University and Research (MIUR), ref. CTN01_00236_683072. The robotic arm (RA) provides the capability to ship a payload and interface with a space tug towards the International Space Station (ISS).

RA is a 7-DOF arm (Figure 2) capable of grasping and moving a payload of $30 \mathrm{~kg}$. It consists of two limbs, each of which includes two intermediate segments, retracted or extended by means of the actuation of limited rotation motors, and seven joints (Figure 3). Each joint is essentially composed of a motor coupled to an epicyclical gear stage, a servo drive with resolver, brake and position sensors.

The robotic arm is locked for the launch and re-entry to the cargo bay of USV3 by means of an hold down system, which consists of two mechanical interfaces with the base of the cargo bay, located respectively in the near of the shoulder joints and at 2/3 of the length of the half limbs. The robotic arm is jointed to the cargo bay base by a dedicated fixture at shoulder joints level (Figure 4). 
This paper is about the evaluation of the loads acting on the robotic arm necessary for its qualification and for the design of its mechanical interfaces with the cargo bay of the spacecraft.

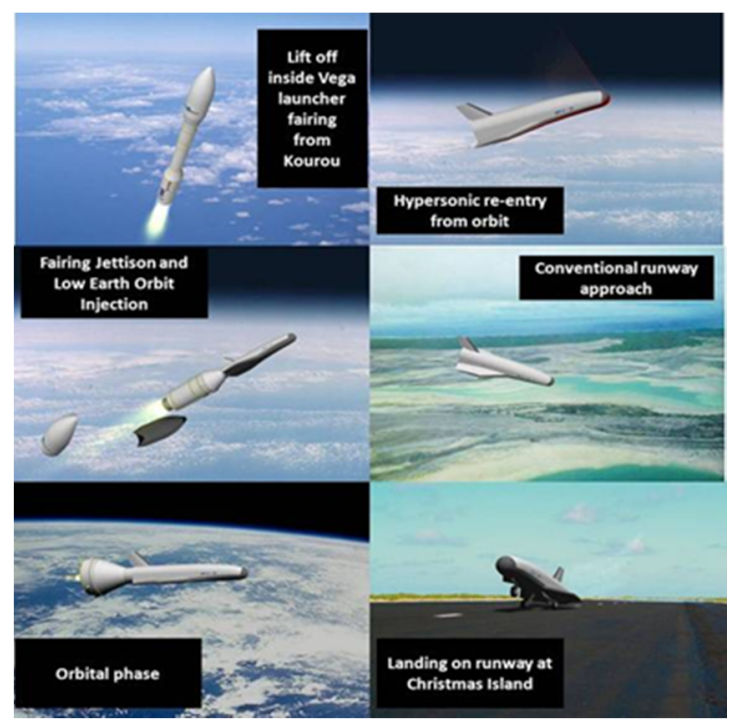

Figure 1: USV3 mission phases.

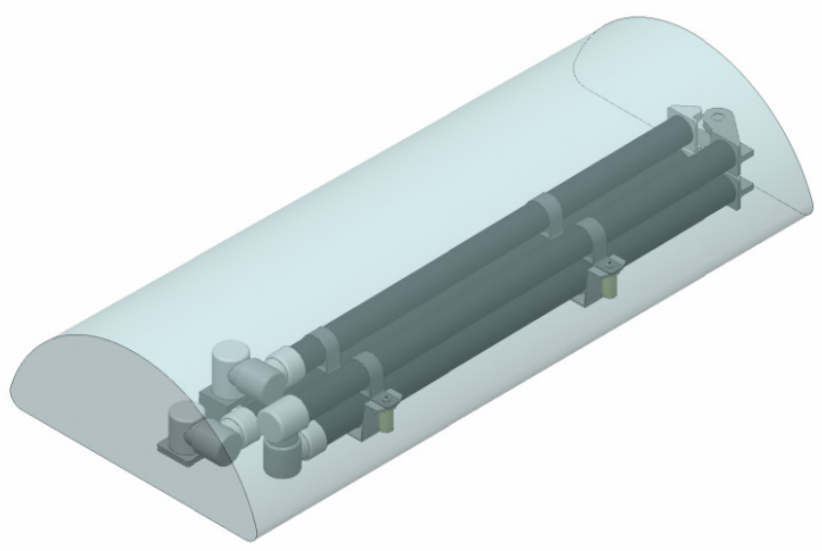

Figure 2: Robotic arm (RA) concept in retracted configuration. 


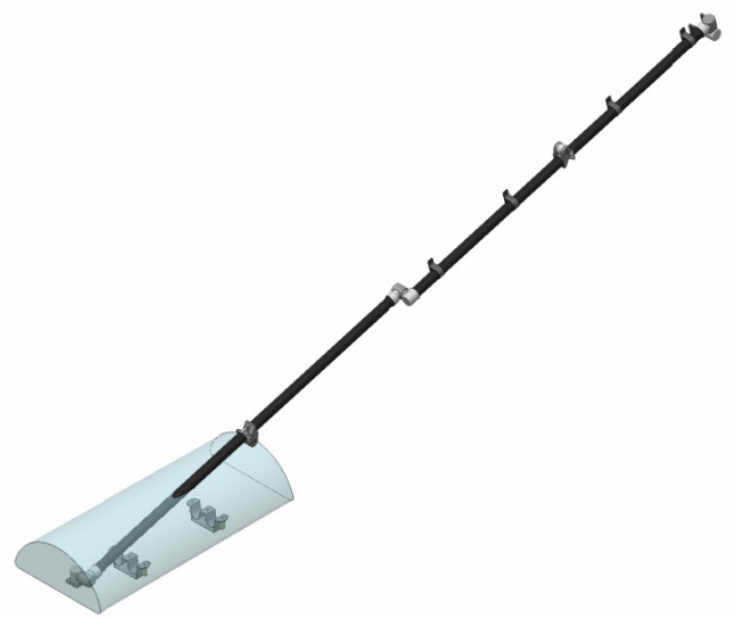

Figure 3: Robotic arm (RA) concept in extended configuration.

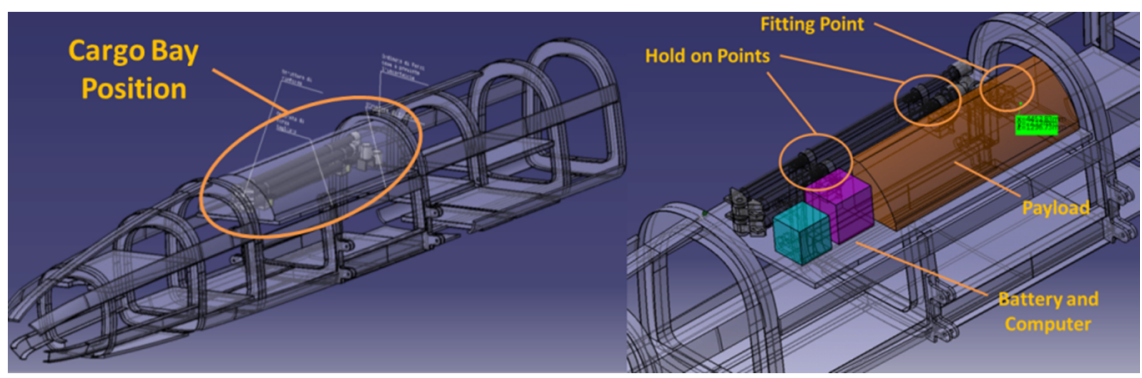

Figure 4: Cargo bay position and equipment arrangement.

\section{Mechanical environment}

During ground operation and flight USV3-PRIDE is subject to different kind of loads, both static and dynamic. These excitations are from different origins such as operational, aerodynamics or propulsion.

Loads at spacecraft to adapter interface are often defined by quasi-static loads (QSL) that apply at spacecraft CoG. These loads are at "system level", thus they are referred to the whole vehicle and do not take into account local higher accelerations due to dynamic phenomena at "subsystem level". VEGA User's Manual [11] gives the limit levels of quasi-static loads to be taken into account for the design and dimensioning of the spacecraft primary structure.

These levels of acceleration are covered by inertia loads coming from the dynamic environment experienced during launch, thus, for the mechanical qualification of the robotic arm and the other subsystems, the dynamic environment provides for most severe conditions in terms of local accelerations. 
The dynamic environment taken under consideration includes two types of dynamic analyses:

- $\quad$ Shock Response Spectrum (SRS) analysis;

- $\quad$ Sine equivalent dynamics.

SRS analysis is necessary to calculate the structural local response to shocks. The spacecraft is subject to shock primarily during stages separations, fairing jettisoning and actual spacecraft separation.

Sine equivalent dynamics accounts for sinusoidal excitations affecting the launch vehicle during its powered flight (mainly the atmospheric flight), as well as during some of the transient phases.

Structural modes are evaluated and dynamic analyses are performed by using the Large Mass Method [12], a well-established methodology which is adopted for a variety of purposes, e.g. seismic analyses of civil engineering structures [13], dynamic response analysis with imposed displacements [14], evaluation of the structural properties of clamped wing for static aeroelastic performance assessment of wind tunnel models [15], and ground load evaluation for unconventional aircraft configurations [16].

With the Large Mass Method a very large mass ( $10^{8}$ times the vehicle mass) is applied at the point of contact of the structure with the ground, allowing to simulate the dynamic response (with a nodal or a modal approach) of a structure integral with the ground and loaded by accelerations coming from the ground itself.

For this kind of application, the large mass is applied to the vehicle fuselage base, that is the location at which the spacecraft is connected to the launcher. All the analyses have been performed by using the MSC.Nastran Software [17].

\subsection{Finite element modelling}

The USV3-PRIDE FE model has been modified to perform dynamic analysis for evaluating local acceleration at the robotic arm centre of gravity.

Figure 5 shows the USV3-PRIDE global FE model. The detail and location of the cargo bay is shown too.

Fe model changes include:

- the fourth frame cut and modification;

- the addition of the cargo bay floor: a constant thickness metallic plate made of titanium alloy has been mated to the three frames by means of 6 RBE2 elements;

- the modelling of subsystems as concentrated masses (CONM2 entry);

- the link of each concentrated mass with the cargo bay floor (RBE3 entry);

- the modelling of the robotic arm fitting to the fifth frame (RBE2 entry). 6 scalar springs are adopted for reading fitting interface loads. 


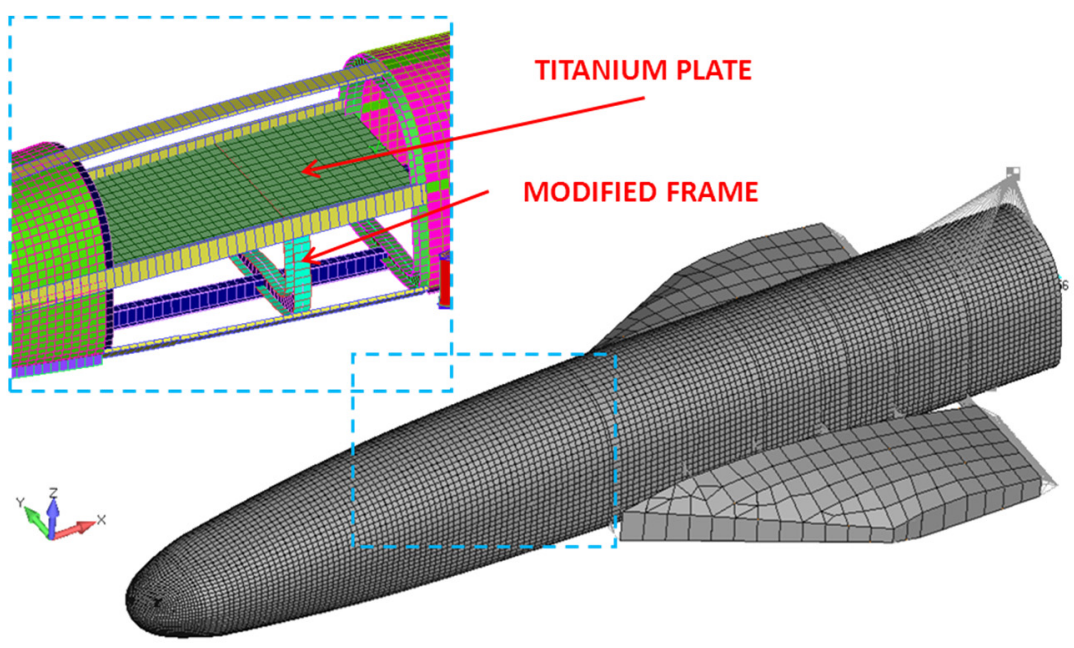

Figure 5: USV3-PRIDE global FE model

Figure 6 shows two different configurations of the robotic arm-payload-cargo bay floor coupling. The first one with robotic arm and payload independently linked to the cargo bay floor, the latter one with the payload integral to the robotic arm, both linked to the cargo bay floor. These two scenarios are foreseen since, at this stage, the possibility to independently link the payload to the cargo bay or alternatively to link the payload to the robotic arm through the grasping system is under investigation.
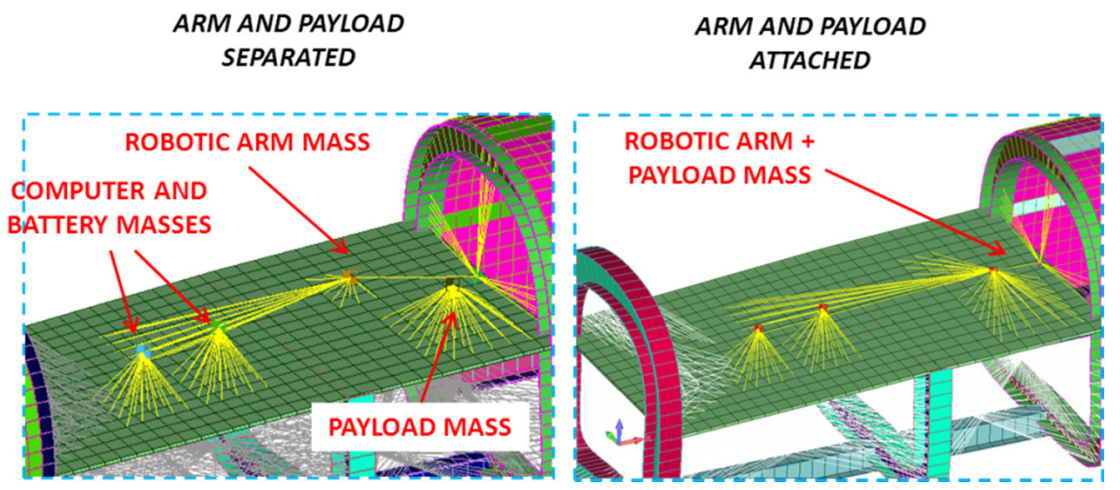

Figure 6: Robotic arm-payload-cargo bay floor coupling.

Anyway, dynamic analyses are performed for both configurations in order to cover all the possible scenarios.

The resulting global FE model mass is compliant with the project mass budget. 
Modal analysis has been performed for the two configurations and shows that flexural and axial first mode frequencies are within the limits imposed by the VEGA User's Manual (frequencies higher than $15 \mathrm{~Hz}$ ).

\subsection{Shock response spectrum}

Shock response spectrum analysis is a methods used to estimate the maximum dynamic response of a structure.

Once known the shock response spectrum at the spacecraft base, which is supplied by [11] and depicted in Figure 7, the analysis consists of a modal analysis of the structure, data recovery, and the response calculation that combines the modal properties of the analysis model with the spectrum data of the applied loads. One crucial aspect of the analysis is the proper choice of a sufficient number of normal modes to represent the entire frequency range of the input excitation and resulting response.

In the particular case of USV3-PRIDE and its robotic arm, the following assumptions have been made:

- $\quad$ Analyses are performed by using MSC.Nastran Sol 103;

- The frequency range of spectrum has been cut at $1000 \mathrm{~Hz}$;

- The modal basis includes rigid modes in the exciting directions and elastic modes up to $1000 \mathrm{~Hz}$, for a total of No. 773 modes;

- A $1.5 \%$ critical damping is used for all modes;

- The vehicle is constrained to its base and it is excited by the acceleration spectrum by using the large mass approach;

- The spectrum is applied simultaneously in radial and longitudinal direction, i.e. in turn along $\mathrm{x}$ and $\mathrm{y}$ axes and along $\mathrm{x}$ and $\mathrm{z}$ axes;

- $\quad$ NRL summation criterion [1].

There are three typical methods to solve a SRS analysis and implemented in the MSC.Nastran software: ABS, SRSS and NLR.

The Absolute Value Method, known as ABS method, assumes the worst case scenario in which all of the modal peak values for every point on the structure occur at the same time and in the same phase. This is very conservative because only a few cycles of each mode typically will occur (e.g. sudden impact). However, in the case of a long term vibration, such as an earthquake when the peaks occur many times and the phase differences are arbitrary, this method could be acceptable.

The square root of sum-squared method, known as SRSS method, sums in vector fashion the results in each direction for each mode first. This is followed by an SRSS calculation for all modes at each selected output quantity $u_{k}$, which is the actual transient response at a physical point. Furthermore it assumes that the modal responses are uncorrelated and the peak value for each mode will occur at a different time. These results are optimistic and represent a lower bound on the dynamic peak values. Usually this method may underestimate the actual peaks. 


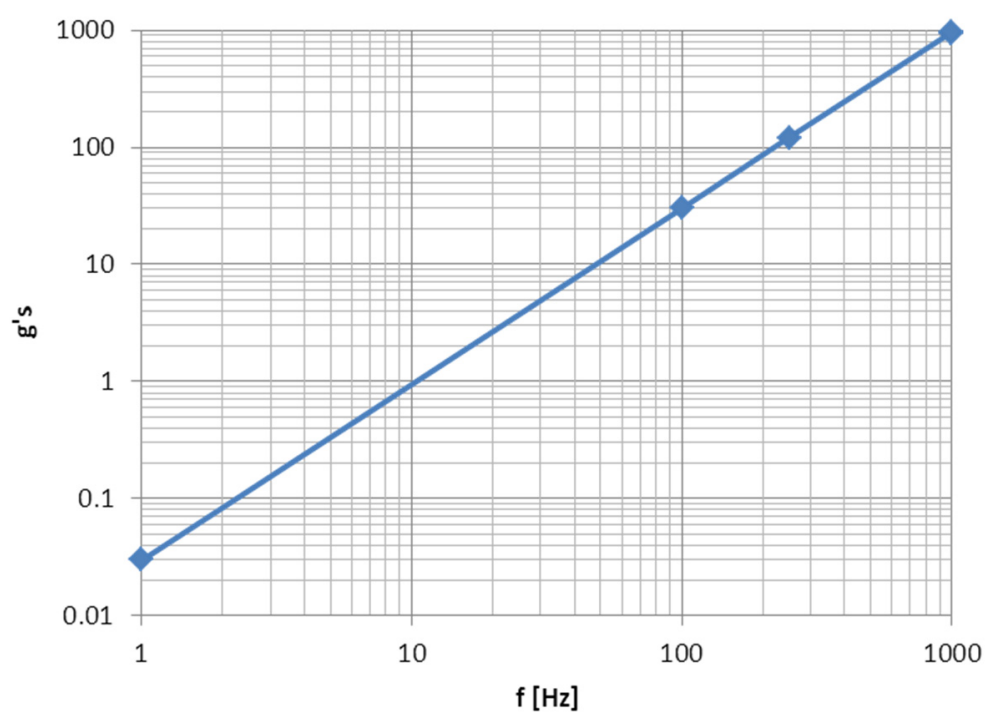

Figure 7: Envelope shock response spectrum.

NRL method was developed by US Naval Research Laboratories. The peak response is given by the equation

$$
\bar{u}_{k} \cong\left|\phi_{j k} \xi_{j}\right|+\sqrt{\sum_{i \neq j}\left(\phi_{i k} \xi_{i}\right)^{2}}
$$

where the $\mathrm{j}^{\text {th }}$ mode is the mode that produces the largest magnitude in the product $\left|\phi_{\mathrm{j} k} \zeta_{\mathrm{j}}\right|$, where $\zeta_{\mathrm{j}}$ is the peak modal magnitude.

The rationale for the method is that the peak response will be dominated by one mode and the SRSS average for the remaining modes could be added directly. The results will fall somewhere between the ABS and SRSS methods.

In the case of VEGA launcher the shock spectrum is applied to the USV3 base exciting all the sub-systems, including the robotic arm.

Results in terms of accelerations (in gs) at the robotic arm CoG are reported for each configuration and for direction of application $\mathrm{x}-\mathrm{z}$ and $\mathrm{x}-\mathrm{y}$.

Table 1 reports acceleration at node 4000005 , which is the center of gravity of the group payload-robotic arm. In this case the payload is not connected to the cargo bay floor, and the robotic arm is connected to the USV3-PRIDE airframe by means of the two hold on points and the fitting. Fitting interface loads are listed in Table 2, they are the action of the robotic arm on the fitting.

Table 3 reports acceleration at node 4000004 , center of gravity of the robotic arm, and at node 3000003 , center of gravity of the payload. In this case the payload is independently connected to the cargo bay floor. Fitting interface loads are listed in Table 4, they are the action of the robotic arm on the fitting. 
Table 1: Acceleration at the robotic arm CoG with integral payload, SRS analysis.

\begin{tabular}{|c|c|c|c|}
\hline \multicolumn{5}{|c|}{ Payload integral to the robotic arm } \\
\hline Reading node \\
\hline 4000005 & 3.501 & -0.085 & 1.260 \\
\hline \multicolumn{4}{|c|}{ Direction of excitation $\mathbf{x}-\mathbf{z}$} \\
\hline $\mathbf{g}$ & $\mathbf{g x}$ & $\mathbf{g y}$ & $\mathbf{g z}$ \\
\hline 81.3 & 72.8 & 27.7 & 23.2 \\
\hline \multicolumn{5}{|c|}{ Direction of excitation $\mathbf{x}-\mathbf{y}$} \\
\hline $\mathbf{g}$ & $\mathbf{g x}$ & $\mathbf{g y}$ & $\mathbf{g z}$ \\
\hline 82.6 & 72.3 & 30.7 & 25.6 \\
\hline
\end{tabular}

Table 2: $\quad$ Interface fitting forces, payload integral to the robotic arm.

\begin{tabular}{|c|c|c|c|c|c|c|}
\hline \multicolumn{7}{|c|}{ Direction of excitation $\mathbf{x}-\mathbf{z}$} \\
\hline $\mathbf{F}_{\mathbf{x}}[\mathbf{N}]$ & $\mathbf{F}_{\mathbf{y}}[\mathbf{N}]$ & $\mathbf{F}_{\mathbf{z}}[\mathbf{N}]$ & $\mathbf{M}_{\mathbf{x}}[\mathbf{N}$ m] & $\mathbf{M}_{\mathbf{y}}[\mathbf{N}$ m] & $\mathbf{M}_{\mathbf{z}}[\mathbf{N}$ m] \\
\hline $5.06 \mathrm{E}+02$ & $3.05 \mathrm{E}+02$ & $3.22 \mathrm{E}+02$ & $1.61 \mathrm{E}+02$ & $7.74 \mathrm{E}+01$ & $3.49 \mathrm{E}+01$ \\
\hline \multicolumn{7}{|c|}{ Direction of excitation $\mathbf{x}-\mathbf{y}$} \\
\hline $\mathbf{F}_{\mathbf{x}}[\mathbf{N}]$ & $\mathbf{F}_{\mathbf{y}}[\mathbf{N}]$ & $\mathbf{F}_{\mathbf{z}}[\mathbf{N}]$ & $\mathbf{M}_{\mathbf{x}}[\mathbf{N} \mathbf{~ m}]$ & $\mathbf{M}_{\mathbf{y}}[\mathbf{N} \mathbf{~ m}]$ & $\mathbf{M}_{\mathbf{z}}[\mathbf{N} \mathbf{~ m ]}$ \\
\hline $5.02 \mathrm{E}+02$ & $3.37 \mathrm{E}+02$ & $3.21 \mathrm{E}+02$ & $2.00 \mathrm{E}+02$ & $7.53 \mathrm{E}+01$ & $3.60 \mathrm{E}+01$ \\
\hline
\end{tabular}

Table 3: Acceleration at the robotic arm CoG, SRS analysis.

\begin{tabular}{|c|c|c|c|c|c|c|c|}
\hline \multicolumn{3}{|c|}{ Reading node (robotic arm) } & \multicolumn{5}{|c|}{ Reading node (payload) } \\
\hline ID & $\mathbf{x}$ & $\mathbf{y}$ & $\mathbf{z}$ & $\mathbf{I D}$ & $\mathbf{x}$ & $\mathbf{y}$ & $\mathbf{z}$ \\
\hline 4000004 & 3.409 & 0.061 & 1.230 & 3000003 & 3.537 & -0.142 & 1.272 \\
\hline \multicolumn{3}{|c|}{ Direction of excitation $\mathbf{x}-\mathbf{z}$} & \multicolumn{5}{|c|}{ Direction of excitation $\mathbf{x}-\mathbf{z}$} \\
\hline $\mathbf{g}$ & $\mathbf{g x}$ & $\mathbf{g y}$ & $\mathbf{g z}$ & $\mathbf{g}$ & $\mathbf{g x}$ & $\mathbf{g y}$ & $\mathbf{g z}$ \\
\hline 115.7 & 62.5 & 29.4 & 92.8 & 119.3 & 49.9 & 67.4 & 84.9 \\
\hline \multicolumn{9}{|c|}{ Direction of excitation $\mathbf{x}-\mathbf{y}$} & \multicolumn{6}{|c|}{ Direction of excitation $\mathbf{x}-\mathbf{y}$} \\
\hline $\mathbf{g}$ & $\mathbf{g x}$ & $\mathbf{g y}$ & $\mathbf{g z}$ & $\mathbf{g}$ & $\mathbf{g x}$ & $\mathbf{g y}$ & $\mathbf{g z}$ \\
\hline 115.8 & 61.7 & 32.9 & 92.3 & 116.8 & 49.2 & 68.6 & 80.8 \\
\hline
\end{tabular}

Sensitivity analyses are performed by varying the input load spectrum. Results show that a spectrum cut below $100 \mathrm{~Hz}$ gives a reduction of acceleration response in $\mathrm{x}-\mathrm{z}$ direction less than $0.8 \%$, thus low frequency elastic modes do not significantly contribute to the total response. 
Table 4: Interface fitting forces, robotic arm with no payload.

\begin{tabular}{|c|c|c|c|c|c|}
\hline \multicolumn{7}{|c|}{ Direction of excitation $\mathbf{x}-\mathbf{z}$} \\
\hline $\mathbf{F}_{\mathbf{x}}[\mathbf{N}]$ & $\mathbf{F}_{\mathbf{y}}[\mathbf{N}]$ & $\mathbf{F}_{\mathbf{z}}[\mathbf{N}]$ & $\mathbf{M}_{\mathbf{x}}[\mathbf{N}$ m] & $\mathbf{M}_{\mathbf{y}}[\mathbf{N}$ m] & $\mathbf{M}_{\mathbf{z}}[\mathbf{N}$ m] \\
\hline $3.68 \mathrm{E}+02$ & $5.34 \mathrm{E}+02$ & $2.11 \mathrm{E}+03$ & $1.66 \mathrm{E}+03$ & $1.82 \mathrm{E}+02$ & $1.00 \mathrm{E}+02$ \\
\hline \multicolumn{7}{|c|}{ Direction of excitation $\mathbf{x}-\mathbf{y}$} \\
\hline $\mathbf{F}_{\mathbf{x}}[\mathbf{N}]$ & $\mathbf{F}_{\mathbf{y}}[\mathbf{N}]$ & $\mathbf{F}_{\mathbf{z}}[\mathbf{N}]$ & $\mathbf{M}_{\mathbf{x}}[\mathbf{N} \mathbf{~ m}]$ & $\mathbf{M}_{\mathbf{y}}[\mathbf{N} \mathbf{~ m}]$ & $\mathbf{M}_{\mathbf{z}}[\mathbf{N} \mathbf{~ m}]$ \\
\hline $3.68 \mathrm{E}+02$ & $5.82 \mathrm{E}+02$ & $2.29 \mathrm{E}+03$ & $1.74 \mathrm{E}+03$ & $1.82 \mathrm{E}+02$ & $1.05 \mathrm{E}+02$ \\
\hline
\end{tabular}

Different modal bases have been used to calculate the acceleration response, from $250 \mathrm{~Hz}$ to $1000 \mathrm{~Hz}$. The difference in the acceleration response are graphically shown in Figures 8 and 9 for the excitation direction $\mathrm{x}-\mathrm{z}$ and both configurations.

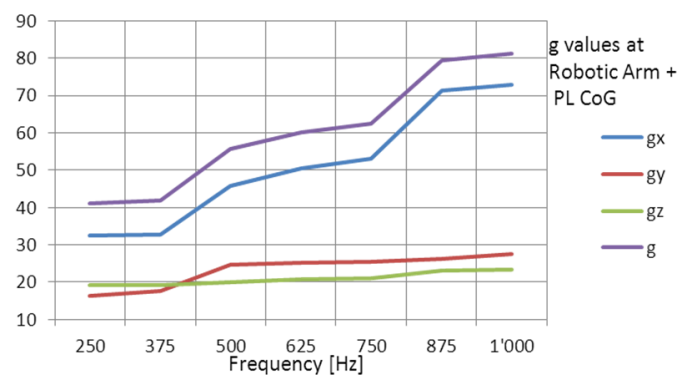

Figure 8: Acceleration response at the RA CoG vs. max frequency modal basis.

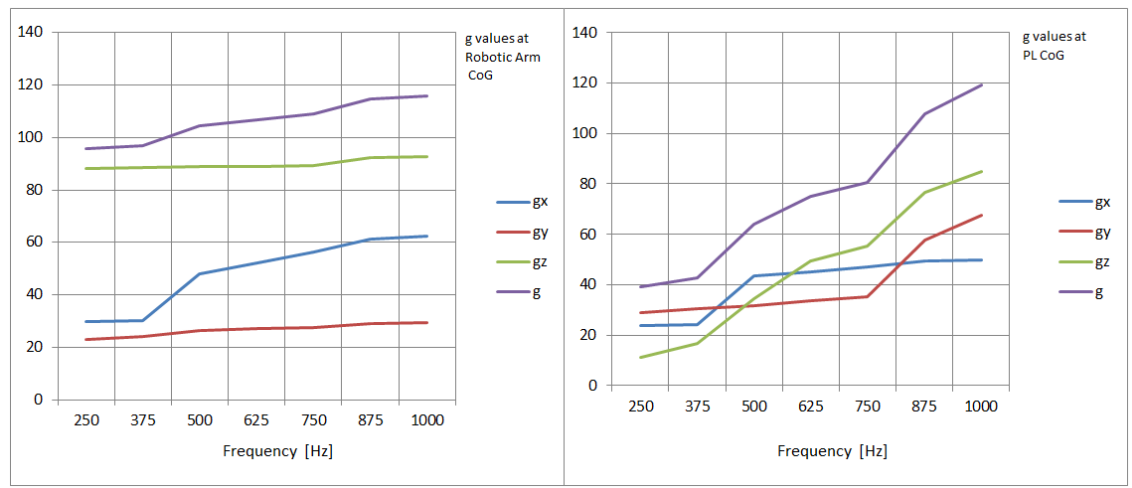

Figure 9: Acceleration response at the RA CoG and at the PL CoG vs. max frequency modal basis.

Referring to Figure 8, $\mathrm{g}_{\mathrm{x}}$ gives the principal contribution to the overall $\mathrm{g}$ value, whereas the contributions along $\mathrm{y}$ and $\mathrm{z}$ directions are much less important. 
As expected, modes having frequency higher than $250 \mathrm{~Hz}$ significantly contribute to the total response. In particular modes between $375 \mathrm{~Hz}$ and $500 \mathrm{~Hz}$ and modes between $750 \mathrm{~Hz}$ and $875 \mathrm{~Hz}$ appear as the most influencing modes. Furthermore, analyses show that enriching the modal basis beyond $900 \mathrm{~Hz}$ does not substantially affect the resulting acceleration response.

Figure 9 shows the sensitivity analysis for the second configuration, with payload and robotic arm independently linked to the spacecraft structure.

For the robotic arm the acceleration response along the $\mathrm{z}$-axis $\mathrm{g}_{\mathrm{z}}$ gives the main contribution whereas $g_{x}$ is responsible of the steep variation between $375 \mathrm{~Hz}$ and $500 \mathrm{~Hz}$.

For the payload, the behavior is similar to the previous configuration, apart from the fact that $g_{z}$ becomes the main component after $500 \mathrm{~Hz}$. Elastic modes under $375 \mathrm{~Hz}$ don't considerably contribute to the overall $\mathrm{g}$ level.

\subsection{Sine equivalent dynamics}

As stated by the VEGA User's Manual, for a spacecraft in single launch configuration, the limit levels of sine-equivalent vibrations at spacecraft-toadapter interface, to be taken into account for the design and dimensioning of the spacecraft, are given in 0 .

Figure 10 shows the same limits in a graphical way.

In the particular case of USV3 and its robotic arm, the following assumptions have been made:

- Analyses are performed by using MSC.Nastran Sol 112 (Modal Transient Analysis);

- The modal basis includes rigid modes in the exciting directions and elastic modes up to $300 \mathrm{~Hz}$, for a total of No. 80 modes;

- The time step used in the analysis is such that there are at least 10 points per period for the higher dynamics to be appreciated $(\mathrm{dt}=3 \mathrm{E}-4 \mathrm{sec})$;

- A $1.5 \%$ critical damping is used for all modes;

- The vehicle is constrained to its base and is excited by the acceleration signal input by using the large mass approach;

- The acceleration signal input is a sine sweep in frequency, with constant acceleration amplitude for each range defined in 0 ;

- The sweep decay is $1 / 2$ oct $/ \mathrm{min}$;

- Sine Vibration inputs are applied separately in longitudinal and lateral directions;

- Acceleration response time-histories are read at the center of gravity of robotic arm and/or payload depending on the configuration. 
Table 5: Sine-equivalent vibrations at spacecraft-to-adapter interface.

\begin{tabular}{|l|c|c|c|c|}
\hline \multirow{2}{*}{ Direction } & \multicolumn{4}{|c|}{ Frequency Band (Hz) } \\
\cline { 2 - 5 } & $1-5$ & $5-45$ & $45-110$ & $110-125$ \\
\cline { 2 - 5 } & \multicolumn{4}{|c|}{ Sine Amplitude (g) } \\
\hline Longitudinal & 0.4 & 0.8 & 1.0 & 0.2 \\
\hline Lateral & 0.4 & 0.5 & 0.5 & 0.2 \\
\hline
\end{tabular}

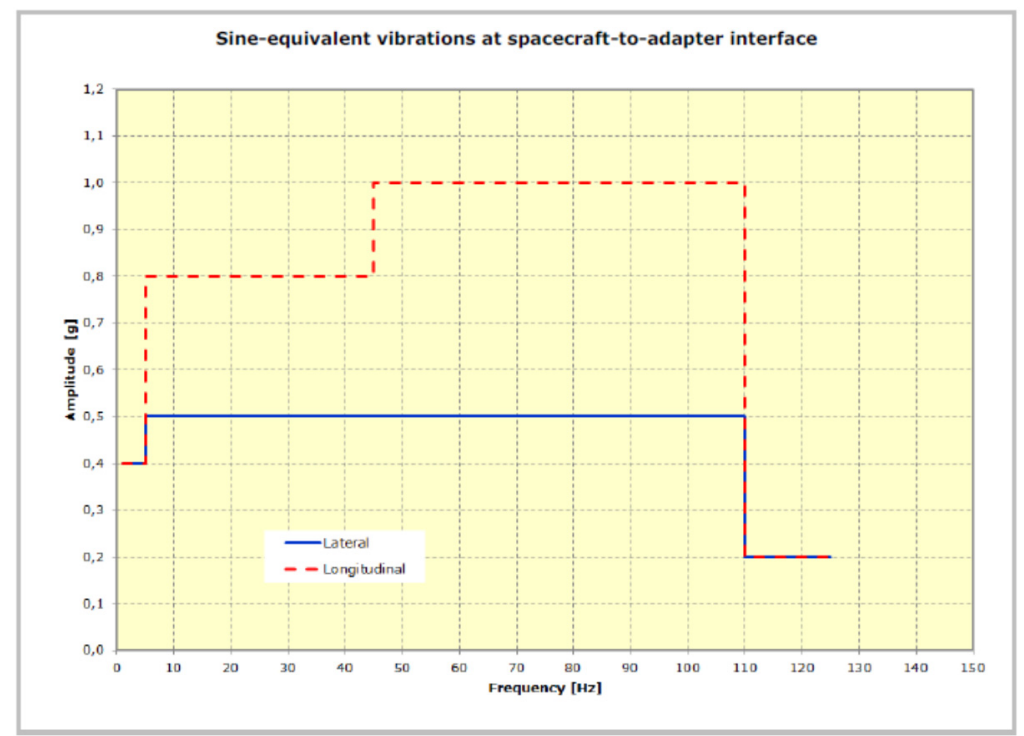

Figure 10: Sine-equivalent vibrations at spacecraft-to-adapter interface.

In the case of VEGA launcher the sine vibration is applied to the USV3 base exciting all the sub-systems, including the robotic arm.

Analysis results show that accelerations at the robotic arm CoG as well as fitting constraint forces, for both configurations, are covered by the SRS results.

Just as an example of sine equivalent dynamics, time histories of $\mathrm{a}_{\mathrm{z}}$ acceleration at the robotic arm $\mathrm{CoG}$ are depicted for both configurations in Figures 10 and 11 , for the range 5-25 Hz and excitation along z-axis.

It is apparent the peak at $15.9 \mathrm{~Hz}$ corresponding to the first flexural mode frequency. 

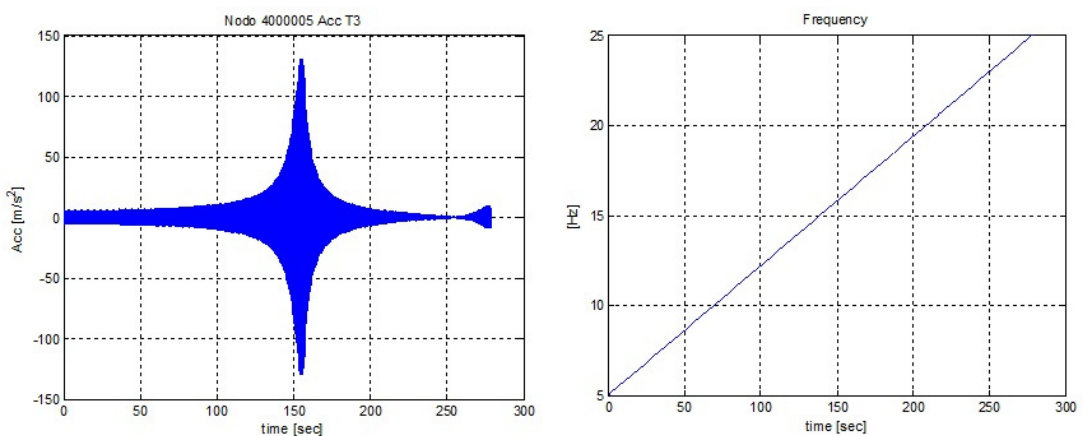

Figure 11: $a_{\mathrm{z}}$ acceleration at the robotic arm CoG, payload integral to the robotic arm.
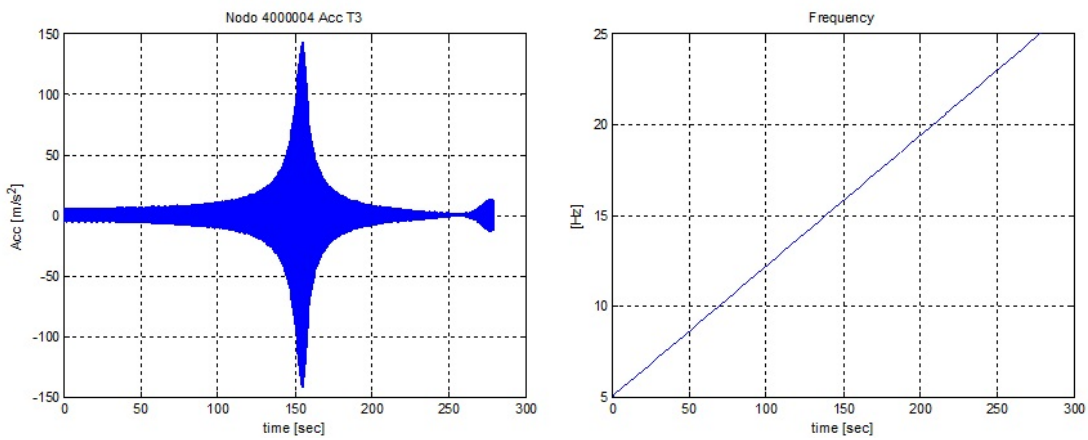

Figure 12: $\mathrm{a}_{\mathrm{z}}$ acceleration at the robotic arm $\mathrm{CoG}$, robotic arm with no payload.

\section{Conclusions}

The evaluation of the dynamic loads for the qualification of a robotic arm, to be installed on-board the USV3-PRIDE spacecraft, has been performed and presented.

Among all kinds of loads exciting the spacecraft during ground operation and flight, for the mechanical qualification of the robotic arm and the other subsystems, the dynamic environment results that providing for the most severe conditions in terms of local accelerations. The dynamic environment taken into account covers the load conditions due to stages separations, fairing jettisoning, actual spacecraft separation (shocks), dynamic excitations affecting the launch vehicle during its powered flight (mainly the atmospheric flight), as well as during some of the transient phases (sine dynamics).

In particular, the structural local response to shocks turns out to be the most critical load condition. 
Qualification loads, provided in terms of local accelerations at the payload CoG for two different configurations, will be used as input body loads for the structural sizing of the robotic arm and its fittings.

\section{References}

[1] Pezzella G., Marini M., Cicala M., Vitale A., Langener T., Steelant J., "Aerodynamic Characterization of HEXAFLY Scramjet Propelled Hypersonic Vehicle", AIAA paper 2014-2844, 32nd AIAA Applied Aerodynamics Conference, AIAA Aviation and Aeronautics Forum and Exposition 2014, Atlanta, GE, USA, June 16-20, 2014.

[2] Di Benedetto S., Rufolo G.C., Marini M., Trifoni E., "European Experimental Reentry Test bed Winglet: From In-Flight Characterization to SCIROCCO Test", AIAA Journal, Vol. 50, No. 10, pp. 2043-2056, October 2012.

[3] Roncioni P., Ranuzzi G., Marini M., Paris S., Cosson E., Walloschek T., "Experimental and Numerical Investigation of Aerothermal Characteristics of Hypersonic Intermediate Experimental Vehicle", Journal of Spacecraft \& Rockets, Vol. 48, No. 2, pp. 291-302, March-April 2011.

[4] Catalano P., Marini M., Nicolì A., Pizzicaroli A., "CFD Contribution to the Aerodynamic Data-Set of the VEGA Launcher", Journal of Spacecraft \& Rockets, Vol. 44, No. 1, pp. 42-51, January-February 2007.

[5] Russo, G., "USV Status 2011:New Steps Ahead", 17th AIAA International Space Planes and Hypersonics and Technologies Conference, San Francisco, USA, 11-14 April 2011, doi:10.2514/6.2011-2242.

[6] De Stefano, M., F., Guidotti, G., Paletta, N., Richiello, C, and Vecchione, L., "USV3: An Autonomous Space Vehicle with Re-Entry and Landing Capability", SAE International 2013-01-2196.

[7] N. Paletta, M. Belardo, D. Lucariello, M. De Stefano Fumo, and U. Mercurio, "The Conceptual Structural Design of an Unmanned Space Vehicle with Re-entry and Landing Capabilities", AIAA 2014-2296, 14th AIAA Aviation Technology, Integration, and Operations Conference.

[8] M. Belardo, N. Paletta, U. Mercurio, "Conceptual Design of the Composite Sandwich Fuselage of a Re-entry Vehicle", ACEX2014.

[9] F. Petrosino, M. De Stefano Fumo, G. Pezzella, P. Catalano, "Evolution of Aerodynamic Shape for a Concept of Autonomous Re-entry Vehicle", $31^{\text {st }}$ AIAA Applied Aerodynamic Conference, 24-27 June 2013, San Diego, CA, USA.

[10] D. Cinquegrana, R. Gardi, V. De Simone, F. Petrosino, P. Catalano, G. Pezzella, M. De Stefano Fumo, "Aerodynamic and TPS Design Analysis of the USV3 Re-Entry Vehicle", 64th International Astronautical Congress, 23-27 September, Beijing, China.

[11] VEGA User's Manual, Arianespace, April 2014.

[12] G. Sitton. MSC/NASTRAN Basic Dynamic Analysis User's Guide, MacNeal-Schwendler Corporation, USA, 1993. 
[13] P. Léger, I. M. Idé and P. Paultre, "Multiple-support seismic analysis of large structures", Computers \& Structures, Volume 36, Issue 6, 1990, pp. 1153-1158, doi: 10.1016/0045-7949(90)90224-P, Copyright (C) 1990 published by Elsevier Science Ltd.

[14] S. Courchesne, A. V.r Popov and R. M. Botez, "New aeroelastic studies for a morphing wing", 48th AIAA Aerospace Sciences Meeting Including the New Horizons Forum and Aerospace Exposition, 4-7 January 2010, Orlando, Florida, USA, AIAA 2010-56.

[15] B.B. Prananta, I W. Tjatra, S.P. Spekreijse, J.C. Kok and J.J. Meijer, "Static aeroelastic simulation of military aircraft configuration in transonic flow", National Aerospace Laboratory NLR, Report No. NLR-TP-2001-346, August 2001.

[16] N. Paletta, M. Belardo, and L. Di Palma, "Non-Linear Dynamic Loads Due to the Landing Impact of a Joined-Wing UAV", SAE 2011 AeroTech Congress \& Exhibition, October 18-21, 2011, Toulouse, France, DOI: 10.4271/2011-01-2720.

[17] MSC.Nastran 2004 Reference Manual.

[18] MSC.Nastran Advanced Analysis User Guide, 2002. 